\title{
Effect of Self Made Buqi Ziyin Recipe Combined with Traditional Chinese Medicine Nursing on the Prognosis Recovery of Patients with Differentiated Thyroid Cancer after Operation
}

\author{
HUI JIE LIANG, C. Y. CHEN, YING LI ${ }^{1}$, XIAO YAN LUO ${ }^{2}$, ZHI XIU ZHAO ${ }^{3}$, G. Y. ZHOU ${ }^{3}$, X. D. WANG ${ }^{3}$, BAO JUAN JIAO $^{4}$, \\ KUANG FEI LIU ${ }^{5}$, CHENG HONG ZHU6 AND RUI ZHANG ${ }^{7 *}$
}

Department of Head and Neck Surgery, Tangshan Gongren Hospital; 'Department of Oncology, Gaoqing County People's Hospital; '2Department of Emergency, Kailuan General Hospital; ${ }^{3}$ Department of general surgery, Qianxi County People's Hospital; ${ }^{4}$ Department of Acupuncture and Moxibustion, Tangshan Anmo Hospital; ${ }^{5}$ Department of Traditional Chinese and Western Medicine Clinical Medicine, Laoting Hospital of Traditional Chinese Medicine; ${ }^{6}$ Department of Nursing, ${ }^{7}$ Department of Health Management Division, PLA Rocket Force Characteristic Medical Center, No. 16 Xinwai Street, Beijing 100045, China

Liang et al::Buqi Ziyin Combined with Traditional Chinese Medicine Nursing on Thyroid Cancer Patients

To explore the effect of self made Buqi Ziyin formula combined with traditional Chinese medicine nursing on the prognosis of patients with differentiated thyroid cancer after operation. 174 patients with differentiated thyroid cancer in our hospital from June 2018 to June 2020 were randomly divided into observation group and control group, 87 cases in each group. The patients in the control group were treated with levothyroxine sodium tablets, 50 100 $\mu \mathrm{g} /$ time once a d, while the patients in the observation group were additionally treated with self made Buqi Ziyin prescription, one dose a day, decoction in morning and evening $200 \mathrm{ml}$ each time. Both groups were treated for $2 \mathrm{mo}$. The control group adopted the routine nursing plan of our hospital, while the observation group added the traditional Chinese medicine meridian massage nursing on the basis of the control group. The immune function, thyroid hormone level, psychological state and treatment effect of the two groups before and after treatment were compared and analyzed. There was no significant difference in the levels of free triiodiothyronine, free thyroxine and thyroid stimulating hormone between the two groups before treatment $(p>0.05)$, which was comparable; after treatment, the levels of free triiodiothyronine and free thyroxine in the observation group were significantly higher than those in the control group and the level of thyroid stimulating hormone in the observation group was significantly higher than that in the control group after treatment, the percentage of cluster of differentiation $3+$ and cluster of differentiation $4+$ in the observation group was significantly higher than that before treatment $(p<0.05)$ and it was also significantly higher than that in the control group after treatment $(p<0.05)$. The percentage of cluster of differentiation $8+$ decreased significantly, which was significantly lower than that before treatment and in the control group $(p<0.05)$ The score was significantly lower than that before treatment $(p<0.05)$; at the same time, it was significantly lower than that in the control group $(p<0.05)$; but there was no statistical difference between the two scores in the control group before and after treatment $(p>0.05)$; after treatment, the total effective rate of the observation group was significantly higher than that in the control group $(p<0.05)$ and the total incidence of complications in the observation group was lower than that in the control group, but the difference was not statistically significant $(\mathrm{p}>\mathbf{0 . 0 5})$. The self made Buqi Ziyin formula combined with traditional Chinese medicine nursing can effectively improve the postoperative recovery speed and quality of life of patients with differentiated thyroid cancer, reduce the probability of disease recurrence, enhance the body's immune ability and reduce the incidence of adverse reactions, which is worthy of clinical application and promotion.

Key words: Differentiated thyroid cancer, self made Buqi Ziyin prescription, traditional Chinese medicine nursing, immune function, thyroid hormone

*Address for correspondence

E-mail: zjghz06@126.com 
Thyroid cancer is the most common malignant tumor in the endocrine system, accounting for $95 \%$ of endocrine malignancies ${ }^{[1]}$. Thyroid cancer ranks first in the incidence of head and neck malignancies in our country ${ }^{[2]}$. Differentiated thyroid cancer (DTC) is a disease with a high prevalence of thyroid cancer and the incidence has been on the rise in recent years ${ }^{[3]}$. Surgical treatment is currently the first choice for the treatment of differentiated thyroid cancer, but patients are prone to various adverse reactions such as longterm anxiety, tachycardia, osteoporosis, etc., due to hormone replacement therapy and psychological stress after surgery ${ }^{[4]}$. It leads to psychological depression and low immune function in patients, which affects the treatment effect and recovery of patient ${ }^{[5,6]}$. Therefore, how to improve the quality of life of patients with thyroid cancer after operation and improve the immune function of the body has become the focus of clinical work.

Traditional Chinese medicine (TCM) believes that the pathogenesis of thyroid cancer can be summarized as "deficiency of both qi and yin and blood stasis". Qi deficiency and yin deficiency are more common and the main evidence is liver depression and stasis ${ }^{[7]}$. Existing experimental and clinical reports have shown that integrated traditional Chinese and western medicine for the treatment of patients with typed thyroid cancer after surgery has advantages in reducing related clinical symptoms, promoting thyroid function recovery and protecting organ function ${ }^{[8]}$. In addition, pain is the most common symptom in postoperative patients and it is also the cause of fear and poor prognosis. At present, more and more TCM meridian and acupoint massage care are used to relieve postoperative pain. At present, there are few reports on the treatment of differentiated thyroid cancer using the treatment plan of Chinese medicine combined with Chinese medicine nursing. Therefore, this study used self made Buqi Ziyin Recipe combined with TCM nursing to treat patients with differentiated thyroid cancer after surgery and explore its influence on patients' postoperative recovery.

\section{MATERIALS AND METHODS}

\section{General clinical data:}

A total of 174 patients with differentiated thyroid cancer admitted to our hospital from June 2018 to June 2020 were selected and randomly divided into observation group and control group, with 87 cases in each group. In the control group, there were 52 males and 35 females, aged 54-73 y, with an average of (61.8 \pm 2.4$)$ $\mathrm{y}$; the disease lasted from 2 mo to $9 \mathrm{y}$, with an average of $(4.7 \pm 1.3) \mathrm{y}$; tumor nodes metastases (TNM) staging $^{[1]}: 28$ cases in stage I, 37 cases in stage $\alpha$ and 22 cases in stage $\beta$. In the observation group, there were 55 males and 32 females, aged 55-76 y, with an average of $(62.7 \pm 2.1) \mathrm{y}$; the disease lasted from 3 mo to $10 \mathrm{y}$, with an average of (4.9 \pm 1.6$) \mathrm{y}$; TNM staging: 30 cases of stage I, 36 cases in stage $\alpha, 21$ cases in stage $\beta$. There was no statistically significant difference between the two groups of patients in general information such as gender, age and course of disease $(p>0.05)$ and they were comparable.

Inclusion criteria-All patients were diagnosed with thyroid cancer by pathological examination, and they were between 50 and 80 y old; Informed consent to this study; Imaging showed no specific lymph nodes in the affected side of the neck and no abnormal lymph nodes in the central group.

Exclusion criteria-Patients with severe primary heart, liver and kidney diseases, hematopoietic system diseases; Patients with other malignant tumors; Patients with thoracic deformities; Allergic constitution or medication contraindications; Pregnancy or breast feeding women; Those suffering from other serious endocrine, circulatory and respiratory diseases; Those who have had previous head and neck radiation therapy. Past thyroid surgery history or with thyroiditis. The tumor has invaded the capsule.

\section{Treatment methods:}

Patients in the control group were given levothyroxine sodium tablets (Germany Merck, Chinese Medicine Zhunzi H20090234, specification: $50 \mu \mathrm{g} /$ tablet) orally, $50-100 \mu \mathrm{g} /$ time, once a day. On the basis of the control group, the observation group took self-made Qi-Ziyin prescription supplemented by TCM nursing and massage of meridian points (Spleen, Zusanli, Yongquan, Qihai, Taixi) and so on. The prescription for nourishing qi and nourishing yin is composed of Taizishen, Ophiopogon japonicus, Schisandra, Polygonatum, Dendrobium, Scrophulariaceae, Fritillaria, Shengdi, Fushen and Roasted Licorice. One dose a day, decocted in water, taken in morning and evening, $200 \mathrm{ml}$ each time. Both groups were treated for 2 mo.

The control group adopts the hospital's conventional nursing plan, while the observation group adds TCM meridian massage care. The details are as follows: the patient is in a prone position, the operator stands on one side, pushes and kneads the back and waist du meridian and bladder meridian with both hands, rolls both sides 
of the spine with the metacarpophalangeal joints and pull the erector spinae with the thumb several on both sides several times and presses Huatuo Jiaji points, press and knead Feishu, Ganshu, Weishu, Shenshu, Dachangshu for $1 / 2 \mathrm{~min}$ each, to dredge the tendons and collaterals, 2 times/d, $10 \mathrm{~d}$ as a course of treatment, a total of six course for nursing.

\section{Observation indexes:}

Evaluation of therapeutic effect-Evaluation criteria for efficacy of TCM syndromes: clinical recovery: TCM clinical symptoms and signs disappear or basically disappear and syndrome scores decrease by $\geq 90$ $\%$; Significant effect: TCM clinical symptoms and signs were significantly improved, syndrome scores decreased $\geq 70 \%$ but $<90 \%$; Effectiveness: TCM clinical symptoms and signs were improved, syndrome score decreased by $\geq 30 \%$ but $<70 \%$; Ineffectiveness: TCM clinical symptoms and signs are not significantly improved or even aggravated and syndrome scores are reduced by $<30 \%$. Total effective rate $=$ (clinical recovery+significant effect+effectiveness)/total number of cases $\times 100 \%$. Thyroid hormone indicators were detected. Before and after treatment, $5 \mathrm{~mL}$ of nonanticoagulant fasting vein blood was collected from the patients. After serum separation, the changes in serum levels of free thyroxine (FT4), thyroid stimulating hormone (TSH) and free triiodiothyronine (FT3) were detected by automatic luminescence instrument; The changes of $\mathrm{T}$ lymphocyte subsets $(\mathrm{CD} 3+, \mathrm{CD} 4+$, CD8+) were detected by flow cytometry; The Hamilton Depression Scale (HAMD ${ }^{[9]}$ and the Self-Rating Depression Scale (SDS) ${ }^{[10]}$ were used to score the patients' depression. The higher the total score, the more serious the depressive state of the patients; the incidence of postoperative complications in the two groups.

\section{Statistical analysis:}

Statistical Package for the Social Sciences (SPSS) 19.0 software was used for data processing and analysis. Measurement data were expressed as $\mathrm{x} \pm \mathrm{s}$ and $\mathrm{t}$ test was used for comparison. Enumeration data were expressed as rate $(\%)$ and comparison was performed by $\chi^{2}$ test. $\mathrm{p}<0.05$ was considered statistically significant.

\section{RESULTS AND DISCUSSION}

The results showed that there were no significant differences in the levels of FT3, FT4 and TSH between the two groups before treatment $(p>0.05)$, which was comparable. After treatment, FT3 and FT4 levels in the observation group were significantly higher than those in the control group, while TSH levels were significantly lower than those in the control group $(\mathrm{p}<0.05)$ and the difference between the two groups was statistically significant, as shown in Table 1.

The results showed that the percentages of CD3+ and $\mathrm{CD} 4+$ in the observation group patients were significantly higher than those before treatment $(\mathrm{p}<0.05)$ and also significantly higher than those in the control group after treatment $(\mathrm{p}<0.05)$, while the percentage of CD8+ was significantly decreased, significantly lower than those before treatment and control group $(\mathrm{p}<0.05)$, as shown in Table 2.

The results of the study showed that the HAMD and SDS scores of the observation group after treatment were significantly lower than those before treatment $(\mathrm{p}<0.05)$; at the same time, they were significantly lower than those of the control group $(\mathrm{p}<0.05)$; while the two scores of the control group before and after treatment were not statistically significant Difference ( $>0.05)$; as shown in Table 3.

TABLE 1: COMPARISON OF THYROID HORMONE LEVELS BETWEEN TWO GROUPS

\begin{tabular}{lccccccc}
\hline Group & Case & \multicolumn{2}{c}{ FT3 $(\mathrm{pmol} / \mathrm{L})$} & \multicolumn{2}{c}{ FT4 $(\mathrm{pmol} / \mathrm{L})$} & \multicolumn{2}{c}{ TSH $(\mu \mathrm{lU} / \mathrm{ml})$} \\
\hline & & Pretherapy & Post-treatment & Pretherapy & Post-treatment & Pretherapy & Post-treatment \\
\hline Observation & 87 & $2.07 \pm 0.17$ & $5.17 \pm 0.64^{\#}$ & $12.77 \pm 3.21$ & $24.35 \pm 3.71^{\#}$ & $4.26 \pm 2.21$ & $1.10 \pm 0.17^{\#}$ \\
Control & 87 & $2.13 \pm 0.21$ & $3.62 \pm 1.15$ & $12.78 \pm 3.33$ & $19.25 \pm 3.56$ & $4.28 \pm 2.25$ & $2.18 \pm 0.21$ \\
\hline
\end{tabular}

Note: "The treatment group was significantly different from the control group after treatment, $\mathrm{p}<0.05$

TABLE 2: IMMUNE FUNCTION PRETHERAPY AND POST-TREATMENT IN THE TWO GROUPS

\begin{tabular}{lccccc}
\hline Group & & Case & CD3 (\%) & CD4 (\%) & CD8 (\%) \\
\hline \multirow{2}{*}{ Observation } & Pretherapy & 87 & $44.68 \pm 8.33$ & $20.53 \pm 5.21$ & $31.63 \pm 4.21$ \\
\cline { 2 - 6 } & Post-treatment & 87 & $64.46 \pm 9.47^{* \#}$ & $47.67 \pm 8.68^{* \#}$ & $20.55 \pm 3.93^{* \#}$ \\
\multirow{2}{*}{ Control } & Pretherapy & 87 & $44.28 \pm 9.65$ & $22.42 \pm 5.32$ & $30.91 \pm 6.25$ \\
& Post-treatment & 87 & $47.51 \pm 8.49$ & $24.61 \pm 4.96$ & $28.41 \pm 5.92$ \\
\hline
\end{tabular}

Note: *Compared with the mean value before treatment in the same group, $\mathrm{p}<0.05$; ${ }^{*}$ Compared with the mean value after treatment in the control group, $\mathrm{p}<0.05$ 
TABLE 3: COMPARISON OF HAMD AND SDS SCORES BETWEEN THE TWO GROUPS OF PATIENTS BEFORE AND AFTER TREATMENT

\begin{tabular}{lccccc}
\hline Group & Case & \multicolumn{2}{c}{ FT3 $(\mathrm{pmol} / \mathrm{L})$} & \multicolumn{2}{c}{ FT4 (pmol/L) } \\
\hline & & Pretherapy & Post-treatment & Pretherapy & Post-treatment \\
\cline { 2 - 6 } Observation & 87 & $32.63 \pm 2.43$ & $18.37 \pm 4.53^{* \#}$ & $64.54 \pm 4.57$ & $38.66 \pm 3.258^{* \#}$ \\
Control & 87 & $31.15 \pm 2.45$ & $29.61 \pm 5.13$ & $65.24 \pm 5.52$ & $60.34 \pm 4.23$ \\
\hline
\end{tabular}

Note: ${ }^{*}$ Compared with the mean value before treatment in the same group, $\mathrm{p}<0.05$; ${ }^{\#}$ Compared with the mean value after treatment in the control group, $\mathrm{p}<0.05$

TABLE 4: COMPARISON OF THERAPEUTIC EFFECTS BETWEEN THE TWO GROUPS

\begin{tabular}{lcccccc}
\hline Group & Case & Clinical recovery & Significant effect & Effectiveness & Ineffectiveness & Total effective rate (\%) \\
\hline Observation & 87 & 53 & 16 & 13 & 5 & $82(94.25)$ \\
Control & 87 & 38 & 21 & 15 & 13 & $74(85.06)$ \\
\hline
\end{tabular}

Note: ${ }^{\#}$ Compared with the mean value of the control group after treatment, $p<0.05$

TABLE 5: THE INCIDENCE OF COMPLICATIONS IN THE TWO GROUPS

\begin{tabular}{lccccc}
\hline Group & Case & $\begin{array}{c}\text { Secondary } \\
\text { hemorrhage }\end{array}$ & $\begin{array}{c}\text { Parathyroid gland } \\
\text { injury }\end{array}$ & $\begin{array}{c}\text { Superior laryngeal nerve } \\
\text { injury }\end{array}$ & $\begin{array}{c}\text { Incidence of total } \\
\text { complications }\end{array}$ \\
\hline Observation & 87 & 3 & 2 & 2 & $7(8.05)$ \\
Control & 87 & 4 & 3 & 2 & $9(10.34)$ \\
\hline
\end{tabular}

Note: \# Compared with the mean value of the control group after treatment, $p<0.05$

The results showed that the total effective rate of the observation group was significantly higher than that of the control group after treatment $(p<0.05)$, as shown in Table 4.

In the observation group, there were 3 cases of secondary hemorrhage, 2 cases of parathyroid gland injury and 2 cases of superior laryngeal nerve injury. There were 4 patients, 3 patients and 2 patients in control group, respectively. The incidence of total complications in the observation group $(8.05 \%)$ was lower than that in the control group $(10.34 \%)$ and there was no statistical difference $(\mathrm{p}<0.05)$, as shown in Table 5 .

At present, ${ }^{131}$ I and levothyroxine sodium are used in clinical treatment for patients with thyroid cancer after surgery. The former killed tumor cells while causing damage to the normal cells of the body; the latter is taken according to individual condition. If the dosage is too high, the patient may have tachycardia, arrhythmia, palpitation, headache and other clinical adverse reactions ${ }^{[1]}$. According to TCM, thyroid cancer belongs to the category of "stone gall" and its pathogenesis is the injury of vital qi caused by Qi stagnation and blood stasis and spittoon coagulation and cementation, which is usually caused by a combination of pathogenic factors such as diet, environment and $\operatorname{mood}^{[12]}$. Surgical treatment of thyroid cancer although removed the focus, but the righteousness has been lost. Insufficiency of qi means that the blood is unable to push and the body presents a state of deficiency of qi and yin, insufficient body fluid or deficiency of yin and yang. On this basis, it is easy to form a syndrome of qi stagnation, phlegm coagulation, blood stasis and 136 internal accumulation. Therefore, in addition to being in a state of deficiency for postoperative patients, recurrence of thyroid nodules can also be $\operatorname{seen}^{[13]}$. Traditional Chinese medicine attributed the adverse reactions caused by levothyroxine sodium treatment after thyroid cancer surgery to the syndrome of yin deficiency and disturbance to the heart. In addition, after the operation, patients with yin deficiency and fire prosperous, deficiency fire on inflammation, yinjin depletion and blood coagulation, most patients will have dry stool, poor sleep palpitations, hoarseness, erosion of the mouth and other clinical symptoms ${ }^{[14]}$. In view of the above pathogenesis characteristics and postoperative symptoms, TCM believes that the basic principles for the treatment of patients with thyroid cancer after surgery should be invigorating spleen qi, nourishing kidney Yin and dispersing toxic compounds. Therefore, this study adopted self made Buqi Ziyin formula combined with TCM nursing symptomatic treatment.

The Buqi Ziyin formula adopted in this research is main composed of Radix pseudostellariae, dwarf lilyturf, Schisandrae chinensis Fructus, rhizoma polygonati, caulis dendrobii, radix scrophulariae, zhejiang fritillary, Shengdi, Fushen, Roasted Licorice. Among them, Taizishen and Schisandra chinensis have the power of nourishing qi and invigorating the spleen. Shengdi, Fushen, Roasted Licorice have the effect of supplementing Qi and warming Yang, Huang Jing, Dendrobium Soothe liver qi, adjust emotions, scattered $\mathrm{Yu}$ Jie. Zhe Fritillaria and Scrophulariaceae can softening hardness to dissipate stagnation, eliminate rmaceutical Sciences 
cancer and detoxify. According to the characteristics of deficiency of vital qi, stagnation of liver qi and existence of residual evil, the patients were treated by syndrome differentiation and overall regulation with definite curative effect. Research data ${ }^{[15]}$ showed that the combination of Radix pseudostellariae and Schisandrae chinensis had a significant effect on deficiency of vital energy, endocrine hypoplasia and decreased immune function in patients with thyroid cancer after surgery and Radix pseudostellariae had a significant effect on serum $T$ cell subsets of tumor patients. Polygonatum and Dendrobium have a definite effect on improving the depression of patients, the anti-inflammatory, sedative and antipyretic functions of Fritillaria and Scrophulariaceae can effectively reduce the adverse stimulation of thyroxine to human organs. This study found that patients in the observation group who took self made Buqi Ziyin Recipe could secrete enough thyroxine for body metabolism when supplemented with TCM massage care and it was significantly better than the control group $(\mathrm{p}<0.05)$, effectively reducing alternative treatment dosage of hormones, at the same time, the lymphocyte subsets CD3+ and CD4+ of the observation group after treatment were significantly higher than the levels before treatment and also significantly higher than the level in the control group after treatment $\mathrm{p}<0.05$ ), while lymphocyte subgroup CD8+ in the observation group after treatment were significantly lower than before treatment, also significantly lower than the control group after treatment $(p<0.05)$, it is suggested that self designed Buqi Ziyin formula can effectively improve the immune function of patients with thyroid cancer after surgery. In terms of treatment effect comparison, the observation group's TCM syndrome score and TCM syndrome efficacy were significantly better than the control group and the incidence of adverse reactions was also significantly better than that of the control group, suggesting the differentiation of combined and nursing treatment for patients with differentiated thyroid cancer after surgery has a good therapeutic effect.

Western medicine mainly uses antidepressant therapy for postoperative depression of malignant tumors, which has a certain effect, but it needs to be taken continuously for a long time and the adverse reactions are obvious ${ }^{[16]}$. Therefore, here are few reports on western medicine therapy for patients with postoperative depression of thyroid cancer. Previous studies have found that the efficacy of TCM in the treatment of depression associated with cancer is comparable to that of Dalixin and the efficacy in the treatment of anxiety associated with Dalixin is superior to that of Dalixin and the side reactions and recurrence are significantly superior to that of Dalixin. In this study, HAMD scores and SDS score of observation group after treatment was significantly lower than before the treatment, indicating that self designed Buqi Ziyin formula combined TCM nursing can effectively relieve depression in the thyroid cancer postoperative patients, to regulate all kinds of adverse reactions from both physical and mental aspects.

In summary, by observing the improvement of clinical symptoms, thyroid function and immune function recovery of the self-made Buqi Ziyin decoction combined with TCM nursing on patients with differentiated thyroid cancer after surgery, it was found that the self made Buqi Ziyin decoction combined TCM care can effectively improve the postoperative recovery speed and quality of life of patients with differentiated thyroid cancer after surgery, reduce the probability of disease recurrence, enhance the body's immune capacity and reduce the incidence of adverse reactions. Therefore, the clinical recovery of patients with differentiated thyroid cancer by using self designed Qi and Yin fimula combined with traditional Chinese medicine nursing is feasible and can be promoted and applied.

\section{Acknowledgement:}

Hui Jie Liang and Chun You Chen have contributed equally to this work; Cheng Hong Zhu and Rui Zhang were considered as co-corresponding authors for this paper.

\section{Conflicts of interest:}

The authors report no conflicts of interest.

\section{REFERENCES}

1. Brown RF, Ducic Y. Aggressive surgical resection of anaplastic thyroid carcinoma may provide long-term survival in selected patients. Otolaryngol Head Neck Surg 2013;148(4):564-71.

2. Conzo G, Polistena A, Calò PG, Bononi P, Gambardella $\mathrm{C}$, Mauriello $\mathrm{C}$, et al. Efficacy of combined treatment for anaplastic thyroid carcinoma: results of a multinstitutional retrospective analysis. Int J Surg 2014;12:S178-82.

3. Denaro N, Nigro CL, Russi EG, Merlano MC. The role of chemotherapy and latest emerging target therapies in anaplastic thyroid cancer. Onco Targets Ther 2013;6:1231-41.

4. Foote RL, Molina JR, Kasperbauer JL, Lloyd RV, McIver B, Morris JC, et al. Enhanced survival in locoregionally confined anaplastic thyroid carcinoma: a single-institution experience using aggressive multimodal therapy. Thyroid 2011;21(1):2530.

5. Goffredo P, Thomas SM, Adam MA, Sosa JA, Roman SA. 
Impact of timeliness of resection and thyroidectomy margin status on survival for patients with anaplastic thyroid cancer: an analysis of 335 cases. Ann Surg Oncol 2015;22(13):416674.

6. Ito KI, Hanamura T, Murayama K, Okada T, Watanabe T, Harada $\mathrm{M}$, et al. Multimodality therapeutic outcomes in anaplastic thyroid carcinoma: improved survival in subgroups of patients with localized primary tumors. Head Neck 2012;34(2):230-7.

7. Lin B, Ma H, Ma M, Zhang Z, Sun Z, Hsieh IY, et al. The incidence and survival analysis for anaplastic thyroid cancer: a SEER database analysis. Am J Transl Res 2019;11(9):5888-96.

8. Mirabile A, Biafora M, Giordano L, Arrigoni G, Cangi MG, Dell'Oca I, et al. Uncommon Site of Metastasis and Prolonged Survival in Patients with Anaplastic Thyroid Carcinoma: A Systematic Review of the Literature. Cancers 2020;12(9):2585.

9. Sun C, Li Q, Hu Z, He J, Li C, Li G, et al. Treatment and prognosis of anaplastic thyroid carcinoma: experience from a single institution in China. PloS one 2013;8(11):e80011.

10. Sykorova V, Dvorakova S, Vcelak J, Vaclavikova E, Halkova $\mathrm{T}$, Kodetova D, et al. Search for new genetic biomarkers in poorly differentiated and anaplastic thyroid carcinomas using next generation sequencing. Anticancer Res 2015;35(4):202936.

11. Tennvall J, Lundell G, Wahlberg P, Bergenfelz A, Grimelius $\mathrm{L}$, Åkerman M, et al. Anaplastic thyroid carcinoma: three protocols combining doxorubicin, hyperfractionated radiotherapy and surgery. Br J Cancer 2002;86(12):1848-53.

12. Thariat JO, Sio TT, Barsela G, Carrie C, Khalifa J, Sun X, et al. Prognosis of irradiated anaplastic thyroid carcinomas: a rare cancer network study. Int J Radiat Oncol Biol Phys 2014;90(1):S549-50.

13. Tiedje V, Stuschke M, Weber F, Dralle H, Moss L, Führer D. Anaplastic thyroid carcinoma: review of treatment protocols. Endocr Relat Cancer 2018;25(3):R153-61.

14. Wächter S, Vorländer C, Schabram J, Mintziras I, Fülber I, Manoharan J, et al. Anaplastic thyroid carcinoma: changing trends of treatment strategies and associated overall survival. Eur Arch Otorhinolaryngol 2020:1-8.

15. Wendler J,Kroiss M, GastK, Kreissl MC,Allelein S, Lichtenauer $\mathrm{U}$, et al. Clinical presentation, treatment and outcome of anaplastic thyroid carcinoma: results of a multicenter study in Germany. Eur J Endocrinol 2016;175(6):521-9.

16. Yau T, Lo CY, Epstein RJ, Lam AK, Wan KY, Lang BH. Treatment outcomes in anaplastic thyroid carcinoma: survival improvement in young patients with localized disease treated by combination of surgery and radiotherapy. Ann Surg Oncol 2008;15(9):2500-5.

This is an open access article distributed under the terms of the Creative Commons Attribution-NonCommercial-ShareAlike 3.0 License, which allows others to remix, tweak, and build upon the work non-commercially, as long as the author is credited and the new creations are licensed under the identical terms

This article was originally published in a special issue, "Evolutionary Strategies in Biomedical Research and Pharmaceutical Sciences" Indian J Pharm Sci 2021:83(3) Spl issue;133-138 